\title{
COMO (QUE) Y SUS FUNCIONES DISCURSIVAS EN EL HABLA SANTIAGUINA: ANÁLISIS PRAGMÁTICO Y SOCIOLINGÜÎSTICO*
}

\author{
COMO (QUE) AND ITS DISCURSIVE FUNCTIONS IN \\ THE SANTIAGO, CHILE SPEECH: PRAGMATIC AND \\ SOCIOLINGUISTIC ANALYSIS
}

\author{
CONSTANZA PANUSSIS LYON \\ Universidad de Chile \\ cpanussis@gmail.com
}

\author{
ABELARDO SAN MARTÍN NÚNEZZ \\ Universidad de Chile \\ asmartin@uchile.cl
}

\section{RESUMEN}

En este artículo se analizan las funciones pragmáticas y la estratificación social de como (que) con valor discursivo, en una muestra estratificada del habla santiaguina; objetivo para el cual se revisó un corpus de 72 entrevistas sociolingüísticas. Los resultados muestran que existen cuatro funciones de la partícula como (que) en el corpus analizado: 1) atenuadora, 2) de relleno o retardataria del decir, 3) aproximativa y 4) marcación de cita, en el mismo orden de prevalencia estadística. El análisis sociolingüístico, por su parte, arroja una asociación entre el uso de como (que) y los factores sociales considerados. Por una parte, su empleo es mayoritario en el discurso de las mujeres en comparación con el de los hombres, en especial, con las funciones atenuadora y de relleno o retardataria del decir. Por otra, el uso de como (que) en tanto marcador del discurso es predominantemente juvenil. De igual forma, se demostró un empleo preeminente en sujetos del grupo medio alto, en cuanto a frecuencia relativa. No obstante, las pruebas de significación estadística sólo ratifican la sensibilidad de los factores sociales sexo y edad de los sujetos.

Palabras clave: Como (que), variación discursiva, pragmática, sociolingüística, español de Chile.

*Este estudio forma parte del proyecto FONDECYT Regular No 1161422, "Los marcadores del discurso en el español hablado en Santiago de Chile: análisis pragmático y sociolingüístico”. Se sintetizan, reformulan y amplían aquí los principales hallazgos de la tesis de licenciatura de Constanza Panussis Lyon, "Análisis pragmático y sociolingüístico de las funciones del marcador como (que) en el habla de Santiago de Chile", investigación dirigida por Abelardo San Martín Núnéz, en el marco del proyecto antes mencionado. 


\section{ABSTRACT}

In this paper the pragmatic functions and the social stratification of como (que) with discursive value were analyzed, in a stratified sample of Santiago's speech; for this purpose, a corpus of 72 sociolinguistic interviews was reviewed. The results show that there are four functions of the particle como (que) in the analyzed corpus: 1) attenuation, 2) filling or retarding, 3) approximate and 4) reported speech marking, in the same order of statistical prevalence. The sociolinguistic analysis shows an association between the use of como (que) and the social factors considered. On the one hand, the use of como (que) is predominant in women's discourse compared to men's, especially with the attenuating and filling or retarding functions. On the other hand, the use of como (que) as discourse marker is predominantly juvenile. Likewise, a pre-eminent use was shown in subjects of the upper middle social group, in terms of relative frequency. However, evidence of statistical significance only ratifies the association with the social factors, sex and age of the subjects.

Keywords: Como (que), discursive variation, pragmatics, sociolinguistics, Chilean Spanish.

Recibido: 16.08.2017. Aceptado: 22.10.207.

\section{INTRODUCCIÓN}

\subsection{Naturaleza, alcance y objetivos}

T a proliferación del empleo de la partícula como (que) en el habla de los chilenos -más allá de sus valores gramaticalmente convencionales, esto es, con un valor atenuador o aproximativo propio de una función pragmática- ha llamado la atención tanto de hablantes como de especialistas (Puga, 1997; Jørgensen, 2012)ํ. Por supuesto, no se trata de un fenómeno exclusivo del español hablado en Chile, ya que se lo ha verificado en otros lugares de habla española tanto en Europa como en América. Específicamente, en el español peninsular el empleo asiduo de como en la conversación, en las décadas de los ochenta y los noventa del siglo XX, fue destacado por autores como Moreno (1992), Vigara (1992) y García y Manjón-Cabeza (1993), entre otros. De hecho, desde el punto de vista normativo, su abuso fue muy criticado por Lázaro (1980 y 1997), quien lo consideraba una "muletilla" innecesaria propia de un hábito inconsciente. En la década más reciente se lo ha atestiguado en el habla juvenil madrileña por Jørgensen y Stemstrøn (2009), Holmvik (2011) y Jørgensen (2012). En América, por su parte, el uso de como en

${ }^{1}$ Es tal la abundancia del empleo de como (que) en el español de Chile actual que resulta tentador denominar "comoqueísmo" a esta tendencia, de acuerdo con el esquema que Rabanales (1974) aplicara para referirse al "dequeísmo", si bien se trata de fenómenos diferentes. De modo semejante, Vigara (1992, pp. 394-395) llama "comismo" al abuso de como en el español peninsular. 
tanto recurso atenuador ha sido descrito por Montes (1980-1981), Puga (1997) y, más actualmente, por Montecino (2004), Jørgensen (2012), Kornfeld (2013) y Puga (2014). En este estudio nos aproximamos al empleo de como (que) en tanto partícula discursiva en el habla de Santiago de Chile, a fin de identificar sus funciones pragmáticas más relevantes y caracterizar su extensión en términos sociolingüísticos. Nuestro objetivo general es analizar las funciones pragmáticas y la estratificación social de la partícula como (que) en un corpus de 72 entrevistas sociolingüísticas realizadas a una muestra estratificada de hablantes santiaguinos. Los objetivos específicos que se desprenden del mismo son: a) identificar las funciones pragmáticas que desempeña dicha partícula en el corpus, b) determinar la frecuencia de uso de estas funciones y c) correlacionar estas frecuencias con las variables sociales sexo-género, edad y grupo socioeconómico de los sujetos de la muestra. A modo de hipótesis, en esta investigación se plantea, por una parte, que como (que) es una partícula discursiva polifuncional y, por otra, que existe una asociación irregular entre su uso y los factores sociodemográficos de los sujetos. La naturaleza del presente estudio es cuantitativa y correlacional y se enmarca en las directrices metodológicas y teóricas aplicadas por San Martín (2004-2005, 2011, 2013,2015 y 2016a y 2016b) para el estudio variacionista de los marcadores del discurso en el español de Chile, en la que se combinan estrategias analíticas de la pragmática y la sociolingüística ${ }^{2}$.

\section{MARCO TEÓRICO}

\subsection{El estudio de como (que) con valor atenuador en la lengua española}

Desde el punto de vista gramatical, Acín (1987) distingue tres funciones de como: adverbio relativo, conjunción y preposición ${ }^{3}$. De un lado, en tanto adverbio relativo, une siempre dos oraciones, donde la subordinada es principalmente modal o comparativa; de otro, en tanto conjunción, vincula un predicado o atributo con un sujeto. Por su parte, como constituye una preposición o "partícula prepositiva" cuando antecede a un nombre o adjetivo para indicar que los conceptos expresados por éstos no deben tomarse en sentido estricto, sino solo de modo aproximado; por ejemplo, "Hicieron una como jaula" (Acín, 1987, p. 27). Es precisamente este último uso descrito, a saber, el denominado como preposicional, el que atañe al presente estudio. Por otra parte, Iglesias (2006) aborda el análisis de esta partícula en tanto marcador del discurso a partir de la gramática de Bello (1988),

\footnotetext{
${ }^{2}$ Una panorámica crítica del estudio de la variación dialectal y sociolingüística de los marcadores del discurso se encuentra en Carbonero y Santana (2010).

${ }^{3}$ Para un mayor detalle del análisis gramatical y discursivo de como en la actualidad, remitimos a Cervera (2009).
} 
quien distingue dos usos prototípicos de como: en términos de adverbio relativo con valor modal y con valores secundarios como el causal, el final o el condicional. Sin embargo, Iglesias (2006) destaca un tercer valor no prototípico de como señalado por Bello, el que se explica a través de transformaciones o cambios en el comportamiento básico anteriormente descrito. Así, presenta lo que Bello (1988, p. 1236) denomina afijo prepositivo: "Empléase también como en calidad de simple afijo o partícula prepositiva, sustituyendo el sentido propio de una palabra o frase el de mera semejanza con él: "Encontró don Quijote con dos como clérigos o estudiantes' (Cervantes)". Su valor, entonces, no es señalar el "contenido propio" de una palabra, frase u proposición, sino el de "semejanza" con él (Iglesias, 2006, p. 818-819). Con respecto a como que, Iglesias explica que para Bello se trata de una variación de su valor prepositivo, destacando que "solo a los verbos o a las proposiciones enteras no puede anteponerse este como sino mediante el anunciativo que" (Bello, 1988, p. 1236). En consecuencia, en este estudio consideraremos a como que como una variante formal de como, debido a que cumplen funciones semejantes (cf. 4, más adelante).

De este modo, algunos lingüistas han destacado que la función de como es, sobre todo, la de atenuar o funcionar como un marcador de parecido no idéntico, lo que permite al hablante proferir un enunciado sin comprometerse con su interpretación literal o exacta. Dentro de esta perspectiva, Montes (1980-1981) analiza diferentes muestras del español de Bogotá, de carácter escrito y oral, a fin de recoger una serie de ejemplos de periódicos, novelas y conversaciones, en los que como se utiliza ya no como adverbio modal, sino que como atenuador (Montes, 1980-1981, p. 667): "Sí, como que he tenido algunas de mi predilección / Estas vacaciones se fueron como ligero, como que no alcanzó el tiempo para nada / Pero sí, me quedé yo como muy desilusionada / Y lo malo es que está como difícil erradicarlo / Se me estaba volviendo como una obsesión". Este autor considera que este uso de como es compartido por la mayoría de los colombianos y que no distingue entre clases sociales; por lo tanto, consiste en un comportamiento general de dicha comunidad de habla; igualmente, interpreta este rasgo atenuador como un "reflejo de cierto apocamiento de las masas colonizadas del nuevo mundo" (19801981, p. 674). Si bien esta última se trata de una interpretación difícil de demostrar, el trabajo de Montes (1980-1981) permite documentar la proliferación del empleo de este como atenuador, ya en los años ochenta, en la variedad de español colombiano. A este respecto, como ya se señala, en varios trabajos se ha destacado el empleo abundante de como con valor atenuador en las postrimerías del siglo XX en el español peninsular. Específicamente, García y Manjón-Cabeza (1993) se aproximan a los nuevos usos de como desde las teorías sintáctica y sociolingüística, en particular, combinando principios de la teoría de las valencias y del paradigma cuantitativo laboviano. Para estos autores, el uso de este como es más frecuente en hablantes jóvenes cultos y tiene un origen madrileño, desde el cual se extendió 
hacia otras ciudades españolas. Además de emplearse en conversaciones, gracias a su carácter culto, penetró en medios de comunicación masiva, en especial, en programas radiofónicos informales. Si bien destacan que hay que guardar una debida prudencia ante este tipo de innovaciones, García y Manjón-Cabeza (1993) lo caracterizan como un estereotipo sociolingüístico del habla juvenil madrileña, que puede interpretarse como un cambio desde arriba (Labov, 1983) de naturaleza casual y propio de la creatividad de los hablantes. No obstante, con posterioridad, García (2015) reporta la desaparición de este como atenuador en la península, calificándolo como un señuelo de la década de los ochenta, que "[...] hoy es un episodio curioso de la historia de la lengua española, un modismo que desapareció de forma tan abrupta como había surgido en el panorama sociolingüístico peninsular" (García, 2015, p. 127).

Debido a su carácter mitigador, en algunos trabajos también se ha incluido a como en el marco de los recursos de atenuación en general. Según Briz (2001 y 2003), la atenuación consiste en una estrategia conversacional que mitiga la fuerza ilocutiva de un acto de habla o la fuerza significativa de una palabra o de una expresión o que le resta importancia a lo dicho; por lo tanto, puede estar asociada a los recursos de cortesía verbal, ya que regula la relación interpersonal y social entre los participantes de un intercambio. Este mismo autor incluye a como dentro de los recursos estratégicos de atenuación (Briz, 2001, p. 144). Asimismo, Puga (1997 y 2014) lo recoge como uno de los recursos léxicos de atenuación característicos de la variedad de habla chilena, concibiéndolo más como un elemento de distanciamiento y mitigación con respecto al mensaje que como una estrategia de cortesía verbal. Montecino (2004), por su lado, lo incluye dentro de los recursos léxicos de atenuación en la conversación de jóvenes universitarios chilenos. Según este autor, como es una partícula de atenuación por modificación externa, mediante la cual un participante "no se responsabiliza de aplicar el contenido de su predicado en toda su intensidad semántica” (Montecino, 2004, p. 23). Por otro lado, Kornfeld (2013) analiza los recursos de atenuación en el español coloquial argentino, entre los que considera a como, atendiendo a los procesos de gramaticalización implicados en su origen y a sus propiedades gramaticales esenciales. De acuerdo con esta autora, los atenuadores rebajan la intensidad del significado de una palabra o una oración y, en tanto estrategia de modalidad, la atenuación también debería estudiarse como parte de la gramática. Si bien no entrega datos concretos, para Kornfeld (2013, p. 23) el empleo de los atenuadores no está distribuido de manera homogénea en el español argentino, siendo el factor etario el determinante, puesto que los utilizan preferentemente los jóvenes.

De manera monográfica, Jørgensen y Stemstrøn (2009) analizan contrastivamente las funciones de like $e^{4}$ en inglés londinense, y de como, en español, utili-

${ }^{4}$ Jørgensen y Stemstrøn (2009) señalan que existe una mayor cantidad de estudios sobre el uso 
zando -para este último- la muestra de español madrileño del Corpus Oral del Lenguaje Adolescente (COLA). Los resultados del contraste entre los marcadores like y como en lenguaje juvenil les permiten a las autoras verificar funciones similares, esto es, atenuación, focalización y citación, con un predominio de la primera, además de determinar que la posición media es la preferente para ambos. Se trata de dos marcadores que son el resultado de procesos de gramaticalización, también con diferenciación social, ya que son más característicos del lenguaje de los jóvenes; de igual modo, like está más extendido en la clase alta y como en el habla de las mujeres. Por su parte, Jørgensen (2012) estudia las funciones de como en el habla de jóvenes de Madrid y de Santiago de Chile, también en las muestras del COLA correspondientes a ambas variedades. Según esta autora, "Como es un marcador discursivo que, como el marcador inglés like, indica parecido no idéntico con lo dicho, permite al hablante decir algo sin comprometerse a una interpretación literal del enunciado" (Jørgensen, 2012, p. 218). Asimismo, destaca la función modalizadora de atenuación de como, tanto en español madrileño como santiaguino, aunque también documenta otras funciones, a saber, intensificador, retardatario del discurso e introductor de discurso directo. También reitera que la posición media de este marcador es la más frecuente. Para Jørgensen (2012, p. 228), existe un contraste social en el uso de este marcador: en Madrid como se emplea más por mujeres jóvenes de clase alta, mientras en Santiago son los hombres jóvenes de clase media quienes más lo hacen. No obstante, como la misma autora concluye, es necesario contrastar el empleo de como en diferentes grupos etarios, aspecto en el que se espera contribuir con el presente estudio, en el que se analiza una muestra estratificada de hablantes santiaguinos, que considera tres grupos de edad de los sujetos.

\section{METODOLOGÍA}

\subsection{Corpus}

El corpus que sirvió de base para este estudio corresponde a 72 entrevistas sociolingüísticas pertenecientes al corpus del grupo de Estudio Sociolingüístico del Español de Chile (ESECH). Las entrevistas fueron realizadas a hablantes santiaguinos, entre 2005 y 2011, por los estudiantes en la asignatura de Sociolingüística de los programas de Licenciatura en Lengua y Literatura Hispánicas y Licenciatura en Lengua y Literatura Inglesas de la Universidad de Chile. En la situación de

de like, los que se remontan a los años ochenta, entre los que sobresale Andersen (2001). También son destacables los trabajos de Tagliamonte (2009 y 2016) sobre be like con la función de marcación de discurso directo en el inglés de hablantes adolescentes. 
entrevista, los entrevistadores debían tratar de superar la "paradoja del observador" consiguiendo, de esta forma, una muestra significativa de discurso natural grabado (estilo vernáculo) de hablantes representativos de la comunidad de habla en estudio (Labov, 1983). La transcripción de las entrevistas antes señaladas fue exhaustivamente revisada, sin exclusión de ninguna de las secciones del instrumento.

\subsection{Población y muestra}

En esta investigación se consideró la población constituida por hombres y mujeres de la Región Metropolitana de más de 20 años de edad. El cuestionario se aplicó a una muestra del tipo por cuotas con afijación uniforme, en la que se divide a la población en estratos o categorías y se asigna una cuota a cada uno de los distintos estratos. La muestra, así conformada, comprende un total de 72 entrevistas realizadas a igual número de sujetos, distribuidos como se indica en la siguiente tabla:

Tabla I. Proporción de sujetos según características sociodemográficas de sexo, edad y grupo socioeconómico.

\begin{tabular}{|l|r|r|r|r|r|r|l|}
\hline & \multicolumn{2}{|c|}{$20-34$} & \multicolumn{2}{c|}{$35-54$} & \multicolumn{2}{c|}{55 y más } & \\
\hline & $\mathrm{H}$ & $\mathrm{M}$ & $\mathrm{H}$ & $\mathrm{M}$ & $\mathrm{H}$ & $\mathrm{M}$ & Total \\
\hline Medio alto & 3 & 3 & 3 & 3 & 3 & 3 & $=18$ \\
\hline Medio & 3 & 3 & 3 & 3 & 3 & 3 & $=18$ \\
\hline Medio bajo & 3 & 3 & 3 & 3 & 3 & 3 & $=18$ \\
\hline Bajo & 3 & 3 & 3 & 3 & 3 & 3 & $=18$ \\
\hline & 18 & 18 & 18 & 18 & 18 & 18 & $=72$ \\
\hline
\end{tabular}

\subsection{Procedimiento de estratificación social utilizado en ESECH}

Para la estratificación de los sujetos que conforman la muestra del estudio, se empleó el sistema de adscripción de estatus social empleado en ESECH que se basa en la asignación del puntaje -que se indica entre paréntesis- a los informantes, de acuerdo con las siguientes variables: a) nivel educacional (3), b) profesión u ocupación (2) y c) comuna de residencia (1). La explicación en detalle de dichas variables se encuentra en San Martín y Guerrero (2015), que consiste en un procedimiento de estratificación basado en determinados estudios acerca de la realidad socioeconómica chilena. A partir de la asignación de los mencionados puntajes, se definieron cuatro grupos socioeconómicos, definidos según los rangos siguientes: 
a) Medio alto (MA): 42 - 36, b) Medio (M): 35 - 27, c) Medio bajo (MB): 26 18 y d) Bajo (B): $17-6^{5}$.

\subsection{Procedimiento analítico}

Nuestro análisis de las funciones discursivas de como (que) consideró la determinación de sus respectivas frecuencias de ocurrencia en las entrevistas que conforman la muestra recopilada. Asimismo, de modo coherente con nuestros objetivos, se observó la incidencia de los siguientes factores sociodemográficos de los hablantes: sexo-género, edad y grupo socioeconómico. El análisis estadístico atendió a dos niveles de observación: a) en términos descriptivos, según frecuencias absolutas y porcentajes de frecuencia y $b$ ) en términos interpretativos o inferenciales, con base en la comparación entre las medias o tendencias centrales ${ }^{6}$.

\section{ANÁLISIS DE RESULTADOS}

\subsection{Análisis pragmático de las funciones de como (que)}

De acuerdo con la clasificación de los marcadores del discurso de Cortés y Camacho (2005), analizaremos, en primer lugar, las funciones interactivas de como (que) y, luego, sus funciones textuales.

\subsubsection{Funciones interactivas de como (que)}

Los marcadores interactivos orientan al oyente sobre las inferencias que debe realizar a propósito de sus relaciones socio-afectivas con el hablante, ya sea centrándose en el interlocutor o en el contenido pragma-lingüístico (Cortés y Camacho, 2005). Las funciones interaccionales de como (que) se sitúan dentro de esta última categoría, ya que, fundamentalmente, matizan el contenido del enunciado.

${ }^{5}$ El intervalo de cada rango responde a la mayor coincidencia con los puntajes que han establecido los estudios sociológicos y de mercado para la clasificación de estratos socioeconómicos, aunque con las debidas adaptaciones, puesto que en esta muestra no se incluyen ni el grupo de la extrema pobreza ni el de la extrema riqueza.

${ }^{6}$ Por lo que se refiere a la descripción de los procedimientos estadísticos aplicados, en este trabajo seguimos las sugerencias e indicaciones de Hernández y Almeida (2005). El paquete estadístico al que hemos recurrido para la estadística inferencial es el SPSS (Statistical Package for the Social Sciences), versión 15.0 para Windows, específicamente, la prueba Análisis de varianza ANOVA. En atención a que la distribución de los datos, en algunos casos, pudiera ser anormal, se complementará dicho análisis con su análogo de tipo no paramétrico, esto es, Anova de Kruskal Wallis. En ambos casos, el grado de significación se definirá en el $5 \%$, según el cual $\mathrm{p}=<0,05$ será estadísticamente significativo. 


\subsubsection{Aproximativa}

Esta función se encuentra descrita en los trabajos sobre like de Andersen (2001) y de como en contraste con like de Jørgensen y Stenstrøm (2009) y Jørgensen (2012). Las autoras sostienen que consiste en aproximar un valor numérico (en lo principal, relacionado con tiempo, distancias o cantidades de diverso tipo) del que no se tiene una certeza absoluta. En este sentido, este valor aproximativo se vincula estrechamente con su función atenuadora, ya que el hablante modaliza su discurso a través de como, dando a entender que la cifra que está entregando es cercana a la cantidad real. La función aproximativa se presentó, prácticamente, en la totalidad de las entrevistas del corpus. A continuación, ilustramos esta función:

1. E: y eso / lo del sueño premonitorio fue / ¿hace cuantos años / tío? I: hace / yo creo ${ }^{7}$ que por lo menos hace como ocho años // muchos años (B II $\mathrm{H} 017)^{8}$.

2. E: ya / ¿hace cuánto tiempo vive en esta comuna? I: yo a esta comuna llevo como vein //a ver // treinta // como treinta años / más de treinta años (B II $\mathrm{H} 019)$.

3. E: ee / bueno / ee ¿hace cuánto vive en esta comuna / sector / o barrio? I: como treinta y siete años < vacilación/> (B II M026).

En los ejemplos 1-3 se observan pausas, vacilaciones y alargamientos (cf. marcas gráficas de la transcripción) que refuerzan el carácter aproximativo (por tanto, inexacto) de las cantidades introducidas por como. Asimismo, en 1 se enriquece

\footnotetext{
${ }^{7}$ Respecto de la transcripción de los ejemplos, creemos necesario indicar las siguientes convenciones gráficas: 1) cuando corresponda a ejemplos de mayor extensión, $\mathrm{E}=$ entrevistador e $\mathrm{I}=$ informante; 2) como se trata de variables no fonéticas y, por lo tanto, la pronunciación no es el foco de nuestro análisis, el texto correspondiente a cada ejemplo se transcribe en ortografía convencional, incluidos los acentos gráficos; 3) con el propósito de no desnaturalizar demasiado la representación del habla chilena, se ha conservado la manifestación gráfica de determinados usos característicos del español hablado en Chile, como el uso del voseo paradigmático con elisión de -s, por ejemplo, estái, soi, viví (estáis, sois, vivís con valor de segunda persona del singular, tú) y el empleo de marcadores del discurso, como po (pues), ¿cachái? (¿entiendes?); 4) para la mejor comprensión de los ejemplos incluidos en este estudio se ha suprimido el sistema de etiquetas pertenecientes al Standard Generalized Markup Language (SGML) contenidas en el Text Encoding Initiative (TEI), que se emplea en la transcripción de las entrevistas de ESECH, excepto las pausas que se señalan con /; 5) se destacaron con subrayado los rasgos prosódicos y léxicos que ayudan a comprender el funcionamiento de la partícula como (que), los que serán explicados en el análisis de los ejemplos. Los rasgos prosódicos destacados en la transcripción son las pausas, las marcas de alargamiento, vacilación y énfasis. La partícula como (que) se presentará siempre destacada en letra cursiva.

${ }^{8}$ El código de los sujetos contiene la siguiente información: grupo socioeconómico $(\mathrm{MA}=$ medio alto, $\mathrm{M}=$ medio, $\mathrm{MB}=$ medio bajo, $\mathrm{B}=$ bajo), grupo etario (III = adultos mayores de 55 años y más, $\mathrm{II}=$ sujetos adultos de edad intermedia entre 35 y 54 años y $\mathrm{I}=$ hablantes jóvenes de entre 20 y 34 años) y sexo $(\mathrm{M}=$ mujer y $\mathrm{H}=$ hombre). A continuación del sexo se indica el número correlativo de cada sujeto en el corpus ESECH.
} 
la intención aproximativa del hablante mediante creo que, predicado doxático, característicamente atenuador, que le permite mitigar la certeza de su enunciado (Haverkate, 1994, p. 123; Montecino, 2004, p. 20). La función aproximativa solo la verificamos para la partícula como y no para su variante como que.

\subsubsection{Atenuadora}

Se trata de la función primordial de como (que) que actúa de manera independiente o en conjunto con el resto de las funciones, a modo de núcleo modalizador. En nuestro corpus, se corrobora que su empleo es predominante en las secuencias argumentativas de las entrevistas; en concreto, en segmentos en los que el hablante da su opinión y busca la aceptación de su interlocutor. De esta manera, como (que) constituye un recurso estratégico que cumple una función mitigadora en el discurso; particularidad inherente de la atenuación lingüística (Briz, 2003, p. 17). Los siguientes ejemplos ilustran la función atenuadora de como (que):

4. I.: no sé po / a mí / puta / con las cosas que he visto de las cabras huevón / así como que son flojas para levantarse / para hacer su aseo personal y todas esas cosas / eeh / eso es lo que me carga / ¡cachái? / porque hay / a las mujeres hay como / hay que moverlas ¡cachái? / y si no las mueves huevón / no / no toman la iniciativa / bueno que también se hacen de querer las huevonas po (B I H003).

5. E: ¿hay algún lugar especial donde te guste ir de vacaciones? I: a mí me gusta la playa// E: ¿por qué? I: porque lo encuentro romántico // o sea lo encuentro romántico pero nunca he ido con ninguna pareja a la playa / pero lo encuentro romántico / no <alargamiento/> es que relaja // la playa relaja / pero no como para estar más de una semana tampoco / porque igual ahora siempre la cosa / hay muchísima gente / entonces como que igual / lo que pasa es que mira / ahora como hay <énfasis> tanta delincuencia <1 énfasis> como que tú andái perseguida / si hay mucha gente tú pensái que te van a robar / que tení que andar mirando pal lado/ entonces todas esas cosas como que a uno <alargamiento/> la asustan / entonces ya <alargamiento/> // un poquito no más /o sea lo justo y necesario (B II M025).

6. E: ¿cuál ha sido el susto más grande de tu vida? o sea que te ha tocado pasar I: eh / no sé o sea yo creo que / me ha pasado me han pasado experiencias con la delincuencia / como que he vivido asaltos y robos / yo creo que por ahí pasa / o sea no ha no ha sido tanto la experiencia sino que es lo que te queda después / como que igual de algún u otro modo me marcaron/ el las experiencias de de de <énfasis> asaltos </énfasis > como que te quedái con $<$ alargamiento/> con <vacilación/> con un con una inseguridad con un miedo que nunca se te val como que queda en ti/ (M I H097). 
Tal como se aprecia en 5 y 6, otra estrategia de atenuación es el distanciamiento del hablante, a través de la desfocalización del centro deíctico, por medio del pronombre de segunda persona tú y del indefinido uno (Haverkate, 1994; Montecino, 2004); recursos que facilitan al hablante generar confianza y solidaridad con sus oyentes, además de atenuar el contenido proposicional con la finalidad de salvaguardar su imagen positiva (Montecino, 2004, p. 27). También, de modo recurrente, como que se combina con la partícula asi (así como que), que conlleva un valor deíctico que refuerza la modalización atenuadora del discurso o con el reformulador de distanciamiento igual (San Martín, 2004-2005); el empleo de ambas partículas acrecienta el carácter atenuante del enunciado introducido por como que.

Por otro lado, con frecuencia, relevamos casos en los que como se combina con algún modalizador de intensificación o cuantificador del tipo muy, mucho, tan, súper, entre otros. Cuando como es seguido por una pausa, Jørgensen y Stenström (2009) y Jørgensen (2012) han descrito que este tipo de construcciones tiene una función intensificadora o enfatizadora, ya que el hablante enfoca o da relieve a información nueva. Para esta interpretación se basan en Fuentes (2008, p. 254), quien señala que el efecto retardatario generado por una pausa en el habla enfatiza el segmento siguiente; este efecto intensificador es también propio del marcador inglés like, según Andersen (2001) (Holmvik, 2011, p. 24). Sin obviar el carácter intensificador que conlleva -en sí mismo- un adverbio cuantificador como los señalados, consideramos que como + cuantificador genera un efecto de atenuación, aun cuando en la transcripción se marque una pausa. Para nosotros se trata de una estrategia de atenuación que tiene como objetivo principal mitigar o suavizar lo dicho, con el fin de proteger tanto la imagen del hablante como la del oyente y evitar ser tan tajante al emitir juicios de valor, ambos efectos vinculados con la cortesía verbal. En este sentido, coincidimos con lo señalado por Landone (2009, p. 324) acerca del uso de como (que). En los siguientes ejemplos se aprecia la atenuación de como + cuantificador:

7. E.: pero ¿nunca quisiste seguir estudiando? / o sea ¿querías trabajar? I.: ee/ en realidad sí quiero seguir estudiando/ pero es que igual quiero juntar <énfasis> plata </énfasis> porque igual nosotros no somos de una familia como muy acomodada entonces digamos que plata para estudiar no tengo (MB I M058).

8. E.: ya / ¿y te gustaba el trabajo? I.: no / no no me gustaba E.: ¿por qué? I.: es que era muy cansador<alargamiento/>y el sueldo era como súper bajo/ pero no me/ me tenía que conformar no más porque/ como está súper difícil encontrar pega/ entonces/ me tuve que conformar con eso y <alargamiento/> y me despidieron (MB I M058).

9. E: ya ¡te ha tocado vivir alguna situación / en donde tu salud estuviera 
comprometida seriamente? I: yo creo que nunca ha sido como tan tan grave como para comprometer mi vida / así al extremo // pero sí hubo un tiempo / digamos / en la media / cuando estuve más o menos mal / cuando me dio la depresión/ (M I M105).

En los ejemplos precedentes es posible elidir como (que) sin que se altere el contenido proposicional del enunciado. Su empleo produce un distanciamiento que mitiga o atenúa el alcance de la aserción. De hecho, esta última, sin la partícula como (que) adquiere mayor fuerza. La neutralización de cuantificadores absolutos como muy mediante el atenuador como también es señalada por Briz (2001, p. 144).

\subsubsection{Marcación de cita en discurso directo}

La función de marcador introductor de cita en discurso directo ha sido considerada tanto para like en inglés, como para como en español. Holmvik (2011, p. 26) y Jørgensen (2012, p. 222) indican que cuando se utiliza como para introducir una cita se destaca que esta última no es literal, esto es, que se reproduce un contenido parecido, no idéntico. A este respecto, es destacable el carácter polifuncional de los marcadores discursivos, ya que, junto con introducir una cita, se está atenuando su contenido, al ser meramente una aproximación. Cabe destacar que también se pueden introducir interjecciones, gestos o pensamientos, al igual que con like (Andersen, 2001, p. 265; Holmvik, 2011, p. 26). A continuación, ejemplificamos el empleo de como para introducir una cita o interjecciones:

10. E: ¿alguna vez has tenido un sueño premonitorio? ¿o algo que hayas soñado y luego haya pasado? I: la verdad es que una situación tan como tan completa no/ pero varias veces he soñado que estoy en alguna parte o en alguna parte de mi casa y después estoy justamente en esa parte y me sorprendo demasiado / o sea como que incluso me llega a dar un poco de miedo /así como "yo estuve aquí juy! esto yo lo soñé" (MB I M057).

11. E.: pero ¿̇tú en el momento lo encontraste dramático? I.: claro po es que en el momento era así como "iguaa!" / mucho escándalo/ (MB I M058).

12. E.: ¿pero tu relación con tus vecinos no es como <alargamiento/> "hola!" / y después lo veí "ah chao!" I.: ah no / ês como "iah oye hola y qué hái hecho!” <alargamiento/> “ ‘ah sí bien!” / (M I H099).

13. I: una vez íbamos en la micro ¿cachái? y yo iba sentada al lado de él po y de repente dobló terrible rápido así y me saqué la chucha huevón y el N / en vez de pararme así me miraba y se cagaba de la risa huevón y todos los pasajeros así como "señora está bien" estaba más roja que </entre risas $>$ // (B I M010). 
El carácter aproximativo de las citas en discurso directo, que rara vez son exactamente literales, se relaciona con su naturaleza básicamente comparativa. Por lo tanto, en la función de introducción de cita se aprecian trazos del valor comparativo original de como. Por otra parte, es destacable la combinación de como con el adverbio así y con el verbo copulativo ser para el cumplimiento de la función de marcación de cita. Esta última combinación ha sido destacada para el inglés be like (Tagliamonte, 2009 y 2016).

\subsubsection{Funciones textuales de como (que)}

Desde una perspectiva textual, los marcadores desempeñan una función de articulación lógico-lingüística entre los segmentos del discurso, por lo tanto, actúan como mecanismos de cohesión y coherencia y reducen, así, el esfuerzo cognitivo del receptor (Cortés y Camacho, 2005, p. 26).

\subsubsection{De relleno y retardataria del decir}

La función textual de como (que) es la de rearticulación de relleno, es decir, dotar a los hablantes de tiempo para formular su discurso; de este modo, en el afán de emitir sus enunciados con fluidez y evitar el silencio, recurren a elementos de apoyo, los que dan la sensación de continuidad. Jørgensen y Stenström (2009) y Jørgensen (2012) también han dado cuenta del valor retardador de como en el discurso oral. En este estudio se considerarán como (que)+<alargamiento $>$, como (que) + pausa, como (que) + como (que), y como (que) intra-segmental como elementos de relleno que funcionan como retardadores, por ejemplo:

14. E.: ¿te han asaltado alguna vez? I.: no / tampoco me han asaltado pero sí he estado como <alargamiento/> siempre ando como con el miedo como que me asalten / es que igual adonde yo vivo igual/ es más o menos // no es muy tranquilo/ (MB I M058).

15. E: ¿recuerdas alguna anécdota / que te haya ocurrido en la víspera / o durante tu matrimonio? I: una anécdota / como que / como que me compré dos ternos / para el matrimonio uno para el civil y el otro para la iglesia / y a mí no me gustan los ternos / yo no uso terno / porque nunca he usado terno / no me gusta no me gusta la corbata encuentro que es un accesorio inútil / más que la corbata no conozco otro (MB II H065).

16. I.: yo creo que bastante ha cambiado porque hace mucha calor / llueve poco / y como que la/ como que por ejemplo yo lo que he notado que hay hay flores que antes en la primavera es como que las flores// ¿cómo se llama? como que las flores se daban antes del tiempo y ahora no/ (MB III M089). 
En síntesis, se identifican cuatro funciones de la partícula como (que): desde una perspectiva interactiva, por un lado, las funciones aproximativa, atenuadora e introductora de cita en discurso directo; desde una perspectiva textual, por otro, la función de relleno y retardataria del decir. Asimismo, se determinó la interacción de unidades léxicas, pragmáticas o rasgos prosódicos que refuerzan cada función de como (que). A continuación, se analizará la incidencia de los factores sociodemográficos en el empleo de las funciones discursivas de como (que).

\subsection{Análisis sociolingüístico de las funciones de como (que)}

En nuestro corpus se identificaron 1.829 casos de empleo de como (que) con las funciones pragmático-discursivas antes señaladas: 1$)$ aproximativa (365 casos con el 20\%), 2) atenuadora (891 con el 48,7\%), 3) marcador de cita (70 con el 3,8\%) y 4) de relleno y retardataria (503 con el $27,5 \%$ ); por consiguiente, se evidencia una preferencia del uso de como (que) con las funciones atenuadora y de relleno y retardataria (ver Gráfico 1):

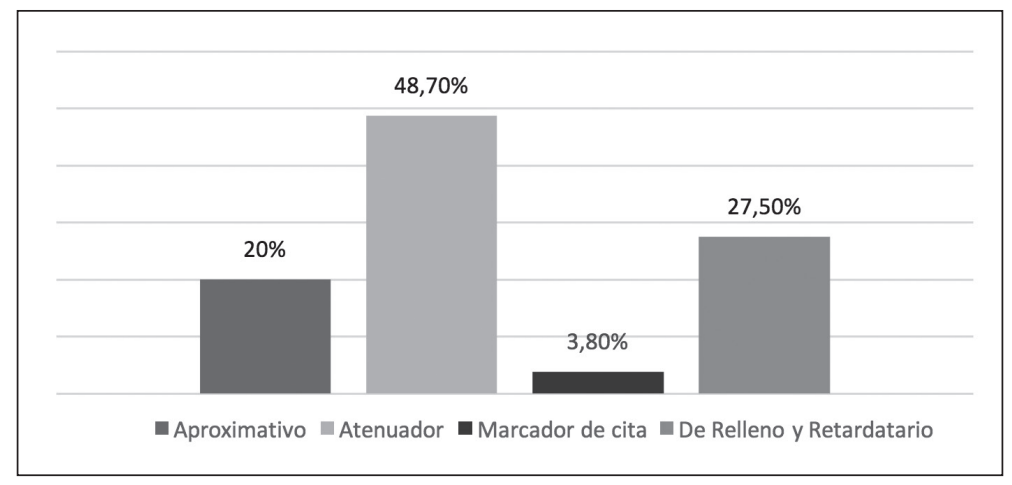

Gráfico 1. Porcentaje de frecuencia de las funciones del marcador como (que) en el corpus ESECH.

Estas frecuencias de empleo se distribuyeron de manera desigual entre los diferentes factores sociales de los sujetos de la muestra, como se verá a continuación.

\subsubsection{Sexo-género}

La Tabla II y el Gráfico 2 exponen las frecuencias absolutas y porcentuales de cada función de como (que), contrastando los resultados entre hombres y mujeres. 
Tabla II. Frecuencia absoluta y porcentual de las funciones de como (que) según sexo-género de los sujetos.

\begin{tabular}{|l|l|c|c|}
\hline Función & Hombres & Mujeres & Total \\
\hline Aproximativa & $184(10,1 \%)$ & $181(9,9 \%)$ & $365(20 \%)$ \\
\hline Atenuadora & $362(19,8 \%)$ & $529(28,9 \%)$ & $891(48,7 \%)$ \\
\hline Marcador de cita & $30(1,6 \%)$ & $40(2,2 \%)$ & $70(3,8 \%)$ \\
\hline De Relleno y Retardatario & $217(11,9 \%)$ & $286(15,6 \%)$ & $503(27,5 \%)$ \\
\hline Total & $793(43,4 \%)$ & $1.036(56,6 \%)$ & $1.829(100 \%)$ \\
\hline
\end{tabular}

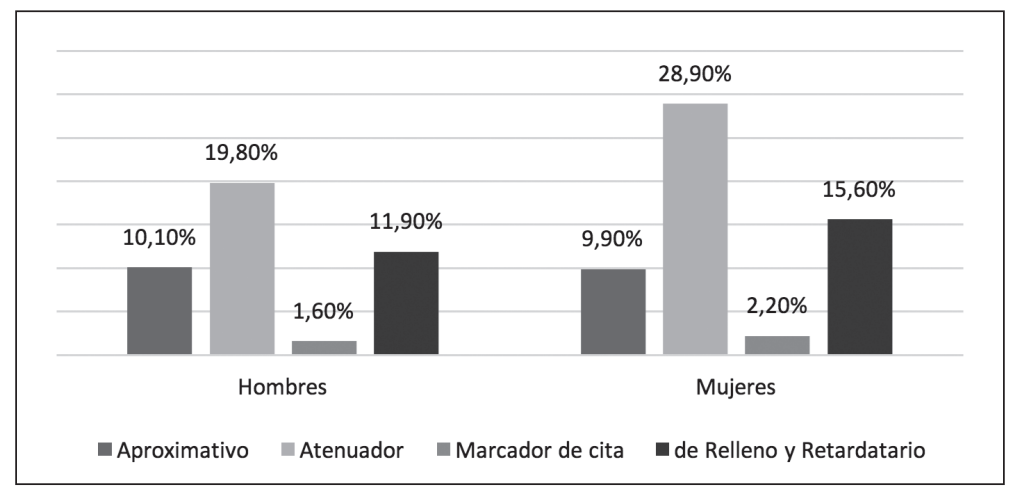

Gráfico 2. Porcentaje de las funciones de como (que) según sexo-género de los sujetos.

Se observa una ligera diferencia en el empleo de como (que) en sus distintas funciones entre hombres y mujeres ( 43,4 y $56,6 \%$, respectivamente). No obstante, este contraste se acentúa si se observa el comportamiento de cada función, debido a un uso mayoritariamente femenino en las funciones atenuadora, marcadora de cita y de relleno y retardataria. Podría explicarse este comportamiento a la luz de los estudios de las diferencias de género en el discurso (Tannen, 1991; Serrano, 2011). A este respecto, Blas Arroyo (2005) destaca que el discurso de las mujeres suele estar más vinculado con la expresión de la emotividad y con una mayor preocupación por su imagen y la de su interlocutor; de igual manera, las mujeres no serían tan tajantes como los hombres, lo que reflejaría marcas de inseguridad en su discurso con mayor frecuencia?.

${ }^{9}$ Con respecto a la función de marcador de cita, San Martín y Guerrero (2013) destacan la prevalencia del empleo del discurso directo en mujeres. 


\subsubsection{Edad}

La Tabla III y el Gráfico 3, por su lado, presentan las frecuencias absolutas y porcentuales de las diferentes funciones de como (que) de acuerdo con la edad de los sujetos de la muestra.

Tabla III. Frecuencia absoluta y porcentaje de las funciones de como (que) según la edad de los sujetos de la muestra.

\begin{tabular}{|l|l|l|l|l|}
\hline Función & $\begin{array}{l}\text { I (20-34 } \\
\text { años) }\end{array}$ & $\begin{array}{l}\text { II (35-54 } \\
\text { años) }\end{array}$ & $\begin{array}{l}\text { III }(55 \text { y } \\
\text { más })\end{array}$ & Total \\
\hline Aproximativa & $147(8 \%)$ & $118(6,5 \%)$ & $100(5,5 \%)$ & $365(20 \%)$ \\
\hline Atenuadora & $385(21 \%)$ & $271(14,9 \%)$ & $235(12,8 \%)$ & $891(48,7 \%)$ \\
\hline Marcador de cita & $50(2,7 \%)$ & $17(0,9 \%)$ & $3(0,2 \%)$ & $70(3,8 \%)$ \\
\hline De Relleno y Retardatario & $236(12,9 \%)$ & $161(8,8 \%)$ & $106(5,8 \%)$ & $503(27,5 \%)$ \\
\hline Total & $818(44,7 \%)$ & $567(31 \%)$ & $444(24,3 \%)$ & $1.829(100 \%)$ \\
\hline
\end{tabular}

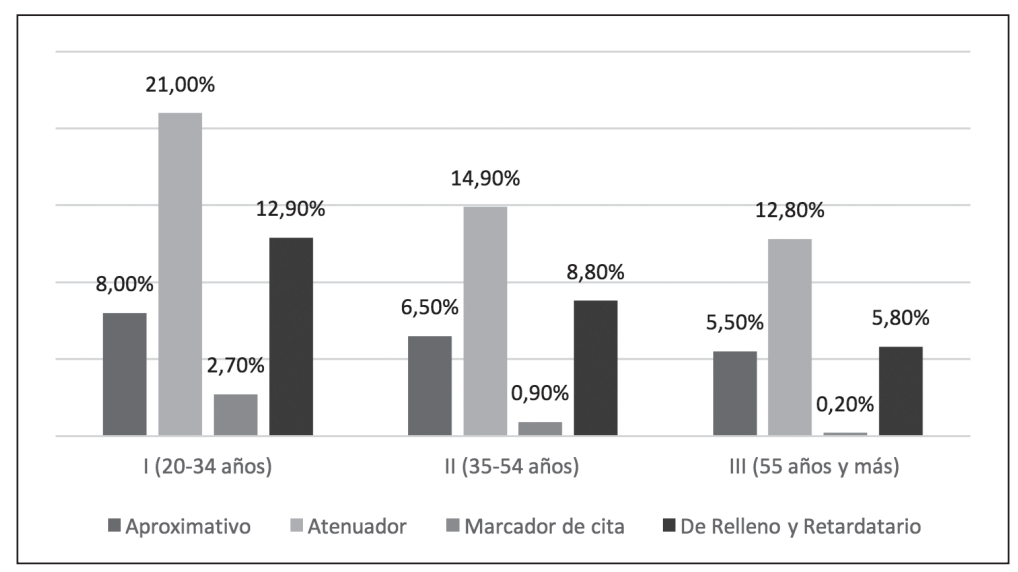

Gráfico 3. Porcentaje de las funciones de como (que) según la edad de los sujetos de la muestra.

Como se ilustra en el Gráfico 3, las frecuencias de empleo de como (que) -en sus cuatro funciones- adoptan un patrón continuo decreciente desde un mayor uso en el grupo etario más joven hasta el más viejo de la muestra. Por lo tanto, 
se puede concluir que se trata de un recurso eminentemente juvenil, que podría explicarse arguyendo una mayor inseguridad o vacilación característica de dicho grupo de edad (Jørgensen, 2012) ${ }^{10}$. Sin embargo, aunque los jóvenes llevan la delantera en el empleo de como (que), nos parece relevante destacar que la cantidad de casos empleados por los grupos de mayor edad no es desdeñable, a excepción de la función de marcador de cita en el grupo de 55 y más años.

\subsubsection{Grupo socioeconómico}

La Tabla IV y el Gráfico 4, finalmente, presentan las frecuencias absolutas y porcentuales de las diferentes funciones de como (que) según el grupo socioeconómico de los hablantes.

Tabla IV. Frecuencia absoluta y porcentaje de las funciones de como (que) según el grupo socioeconómico de los sujetos de la muestra.

\begin{tabular}{|l|l|l|l|l|l|}
\hline Función & Bajo & $\begin{array}{l}\text { Medio } \\
\text { Bajo }\end{array}$ & Medio & Medio Alto & Total \\
\hline Aproximativo & $106(5,8 \%)$ & $106(5,8 \%)$ & $81(4,4 \%)$ & $72(4 \%)$ & $365(20 \%)$ \\
\hline Atenuador & $138(7,5 \%)$ & $195(10,7 \%)$ & $227(12,4 \%)$ & $331(18,1 \%)$ & $891(48,7 \%)$ \\
\hline Marcador de cita & $10(0,4 \%)$ & $12(0,7 \%)$ & $23(1,3 \%)$ & $25(1,4 \%)$ & $70(3,8 \%)$ \\
\hline De Relleno y Retardatario & $120(6,6 \%)$ & $124(6,8 \%)$ & $105(5,7 \%)$ & $154(8,4 \%)$ & $503(27,5 \%)$ \\
\hline Total & $374(20,5 \%)$ & $437(23,9 \%)$ & $436(23,8 \%)$ & $582(31,8 \%)$ & $1.829(100 \%)$ \\
\hline
\end{tabular}

${ }^{10}$ A este respecto, resulta oportuna la siguiente interpretación de Jørgensen (2012, p. 209) en la que, haciendo extensiva la apreciación de Andersen (2001) sobre like, explica el carácter eminentemente juvenil de como (que):

La general inseguridad que caracteriza la etapa entre la niñez y la madurez, lleva a los jóvenes a tomar la palabra, en ocasiones, sin saber qué decir. Se entiende, pues, el uso frecuente de como, ya que la aportación del marcador del discurso como a un enunciado es la de señalar que entre lo dicho y el pensamiento de fondo no hay una total correspondencia, sino una relación de cierto parecido. Permite al hablante decir algo sin comprometerse a una interpretación literal del enunciado.

Sin embargo, las diferencias entre grupos etarios se verifican contrastando, efectivamente, conjuntos de sujetos con edades diferentes, que es lo que realizamos en el presente estudio. 


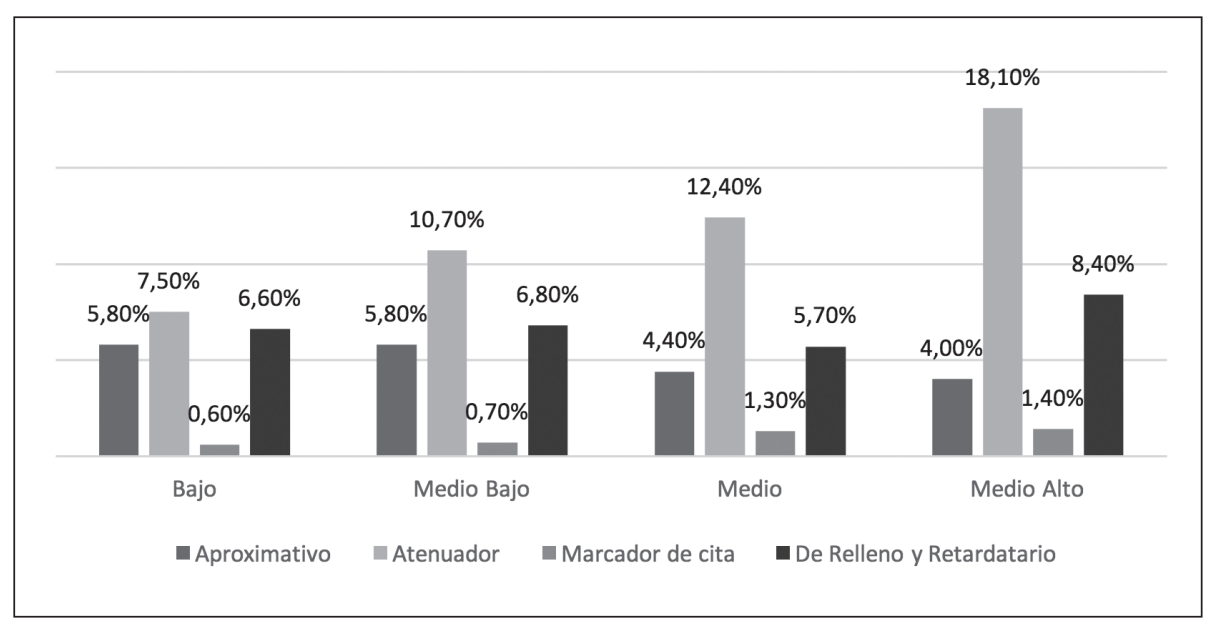

Gráfico 4. Porcentaje de las funciones de como (que) según el grupo socioeconómico de los sujetos de la muestra.

Se observa que existe cierta homogeneidad en las funciones aproximativa, marcación de cita y de relleno y retardataria entre los grupos socioeconómicos considerados en la muestra. Sin embargo, identificamos un patrón curvilíneo creciente en la función atenuadora que es más empleado por los sujetos del grupo medio alto. En consecuencia, por lo que refiere a dicha función, podríamos entender la extensión de como (que) como un cambio desde arriba (Labov, 1983), puesto que se da mayormente en el grupo de mayor prestigio (Moreno, 2009, p. 114), por lo menos, en términos estadísticos descriptivos.

\subsection{Análisis estadístico inferencial}

Los resultados del análisis estadístico inferencial del total de casos de como (que) incluyendo sus cuatro funciones arrojó significación estadística para el contraste de medias de las variables sexo-género y edad de los sujetos, pero no para el grupo socioeconómico. Por una parte, la prueba ANOVA dio los siguientes resultados para el primero de los mencionados factores: $F=11,368$ y $p=0,001$. Las medias correspondientes fueron: 22,000 para los hombres y 28,806 para las mujeres (ver Gráfico 5). De igual modo, Anova de Kruskal-Wallis confirmó dicha significación estadística (Chi-cuadrado=4,656 y p=0,031). 


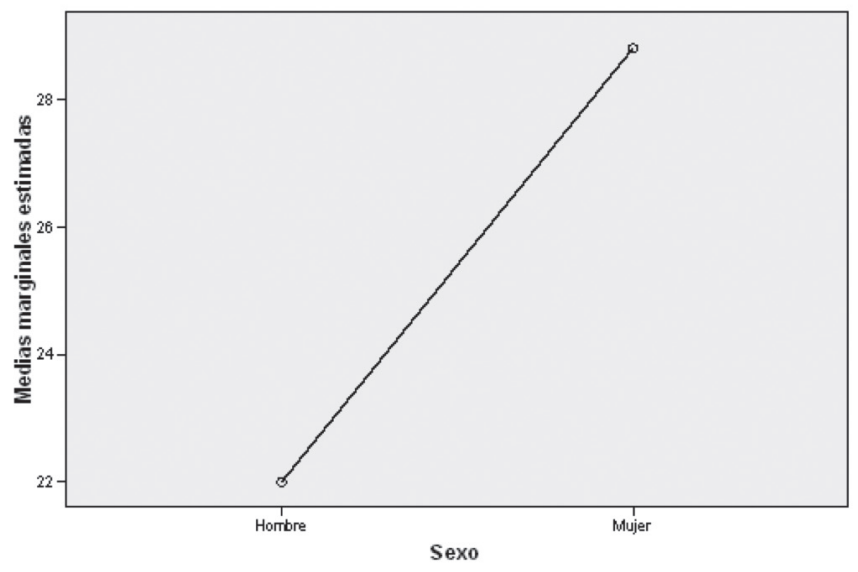

Gráfico 5. Medias marginales estimadas del total de como (que) según el sexo-género de los sujetos.

Por otra parte, el análisis de la varianza aplicado a la variable edad proporcionó los resultados que siguen: $\mathrm{F}=20,715 \mathrm{y} \mathrm{p}=0,000$. Las medias para este factor se ilustran en el Gráfico 6: 34,083 para el grupo I (20-34 años), 23,667 para el grupo II (35-54 años) y 18,458 para el grupo III ( 55 años y más). Igualmente, la prueba no paramétrica ratificó la significación de este factor (Chi-cuadrado=17,337 y $\mathrm{p}=0,00)$.

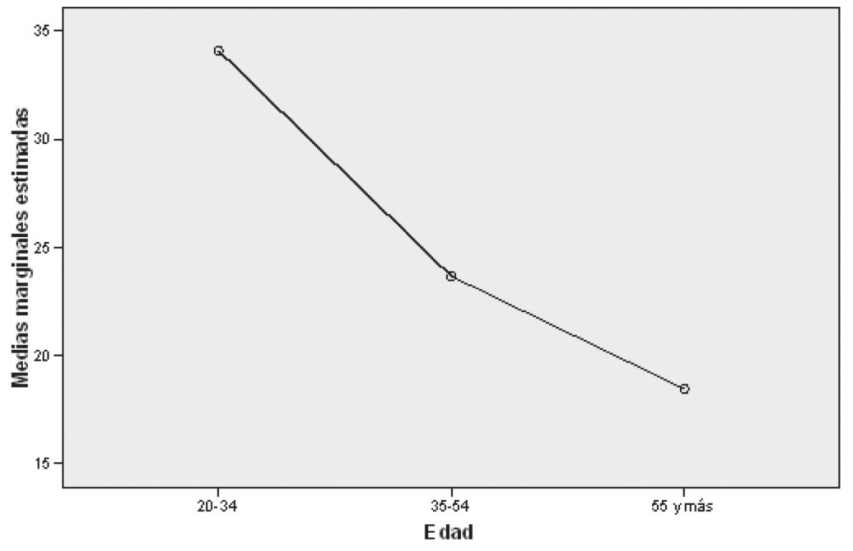

Gráfico 6. Medias marginales estimadas del total de como (que) según la edad de los sujetos.

En consecuencia, este análisis estadístico permitió confirmar la sensibilidad de los factores sexo-género y edad en el empleo de como (que), en tanto partícula 
discursiva, la que es más frecuente en mujeres y en sujetos jóvenes.

\section{CONCLUSIONES}

En este trabajo se analizó las funciones pragmáticas y la estratificación social de como (que), con valor discursivo, en un corpus de 72 entrevistas sociolingüísticas, que corresponden a una muestra del español hablado en Santiago. Las conclusiones más significativas de este artículo pueden sintetizarse de la siguiente forma:

1. En el corpus se identificó 1.829 casos de empleo de como (que), en tanto partícula discursiva, con las siguientes funciones: atenuadora (891, 48,7\%), de relleno y retardataria $(503,27,5 \%)$, aproximativa $(365,20 \%)$ y marcadora de cita $(70,3,8 \%)$.

2. Desde el punto de vista pragmático, la partícula como (que) se da preferentemente en secuencias de discurso de tipo argumentativo, en particular, cuando el hablante está dando su opinión sobre algún tema. Su función atenuadora se relaciona con segmentos de discurso en los que se ve comprometida la imagen del hablante con respecto al oyente, por lo que su estudio se enmarca dentro del ámbito de la cortesía verbal (Landone, 2009). La atenuación constituye la función nuclear de como (que), de la cual se derivan las funciones aproximativa y marcación de discurso directo. La función de relleno y retardataria del decir permite a los hablantes dar fluidez o continuidad a su discurso, planificando lo que se quiere decir con mayor precisión. Todas estas funciones, a menudo, son reforzadas mediante recursos léxicos y prosódicos. Por lo tanto, comprobamos la primera hipótesis de nuestro estudio, a saber, que como (que) es una partícula discursiva polifuncional.

3. Desde el punto de vista sociolingüístico, en términos estadísticos descriptivos, existe una asociación entre el uso de la partícula como (que) y los factores sociodemográficos de los sujetos. Se comprobó que el uso de como (que) es mayor en las mujeres $(56,6 \%)$ que en los hombres $(43,4 \%)$, en particular, en las funciones atenuadora $(30 \%)$ y de relleno y retardataria $(15,6 \%)$. Asimismo, los datos muestran que la utilización de la partícula como (que) es predominantemente juvenil (44,7\%). También se obtuvieron resultados interesantes al correlacionar el factor social grupo socioeconómico con la partícula como (que). Igualmente, las funciones de atenuación $(18,8 \%)$, de relleno y retardataria $(8,4 \%)$ y marcación de cita $(1,4 \%)$ se presentan mayormente en el grupo socioeconómico medio alto, por lo que podríamos entender la proliferación del marcador como (que) como un cambio en marcha desde arriba. Con respecto a la aplicación de pruebas de estadística inferencial, se confirmó la significación estadística de los factores sexo-género y edad, pero no la del factor grupo socioeconómico, 
esto es, que el mayor empleo de como (que) está asociado con las mujeres y con hablantes jóvenes. De este modo, corroboramos la segunda hipótesis de este trabajo, es decir, que existe una asociación irregular entre el empleo de la partícula como (que) y los factores sociodemográficos de los sujetos.

4. A nuestro juicio, como (que) es una partícula en vías de gramaticalización, ya que aún conserva parte de su significado como adverbio comparativo, principalmente en las funciones aproximativa, atenuadora y marcación de cita. $\mathrm{Su}$ uso más frecuente consiste en atenuar un enunciado, así como dar tiempo al hablante para re-articular su discurso. Se trata de una partícula modalizadora cuyo empleo ha proliferado notablemente en el habla juvenil; en especial, en mujeres del grupo socioeconómico medio alto, pero que está extendiendo su empleo a hablantes más adultos, a los hombres y a sujetos de otros grupos socioeconómicos. En este sentido, sería interesante observar en el futuro si los hablantes que hoy son jóvenes, al pasar a la adultez mantienen este comportamiento o si, por el contrario, la partícula como (que) desaparece paulatinamente de su discurso.

\section{REFERENCIAS}

Acín, Esperanza. 1987. Construcciones con "como" en español moderno. RILCE, 3(1), 25-59.

Andersen, Gisle. 2001. Pragmatic markers and sociolinguistic variation. A relevancetheoretic approach to the language of adolescents. Amsterdam/ Philadelphia: John Benjamins.

Bello, Andrés. 1988. Gramática de la lengua castellana destinada al uso de los americanos. Con notas de Rufino José Cuervo, estudio y edición de Ramón Trujillo. Madrid: Arco libros.

Blas Arroyo, José Luis. 2005. Sociolingüistica del español. Desarrollos y perspectivas en el estudio de la lengua española en contexto social. Madrid: Cátedra.

Briz, Antonio. 2001. El español coloquial en la conversación. Esbozo de pragmagramática. Tercera edición. Barcelona: Ariel.

Briz, Antonio. 2003. La estrategia atenuadora en la conversación cotidiana española. En D. Bravo (ed.), Actas del Primer Coloquio del Programa EDICE, 17-46. Disponible en http://www.edice.org/descargas/1coloquioEDICE.pdf (Consulta 01/06/2016).

Carbonero, Pedro y Santana, Juana. 2010. Marcadores del discurso, variación dialectal y variación social. En Ó. Loureda y E. Acín (coord.), Los estudios sobre marcadores del discurso en español, hoy. Madrid: Arco/Libros, 497-521.

Cervera, Ángel. 2009. Tratamiento gramatical y comportamiento discursivo de como en el español actual. Español Actual, 91, 57-98. 
Cortés, Luis y Camacho, María. 2005. Unidades de segmentación y marcadores del discurso. Madrid: Arco/Libros.

Fuentes, Catalina. 2008. La aproximación enunciativa. Lingüistica Española Actual, 30(2), 223-258.

García, Francisco. 2015. Sociolingüistica. Madrid: Editorial Síntesis.

García, Francisco y Antonio Manjón-Cabeza. 1993. Teoría sintáctica y cambio sociolingüístico (a propósito de los nuevos usos de como en español). Docencia e Investigación: revista de la Escuela Universitaria de Magisterio de Toledo, 3, 35-59.

Haverkate, Henk. 1994. La cortesía verbal en español. Madrid: Gredos.

Hernández, Juan Manuel y Manuel Almeida. 2005. Metodología de la investigación sociolingüística. Málaga: Editorial Comares.

Holmvik, Lise. 2011. Como usado como marcador del discurso en el lenguaje juvenil de Madrid. Tesis de Máster del Departamento de Lenguas Extranjeras. Bergen, Noruega: Universidad de Bergen.

Iglesias, Manuel. 2006. Una vez más, Bello: Como y los marcadores del discurso. En A. Roldán Pérez (coord.), Caminos actuales de la historiografía lingüistica: actas del V Congreso Internacional de la Sociedad Española de Historiografía lingüistica, Vol. 2, 815-832.

Jørgensen, Annette. 2012. Funciones del marcador pragmático como en el lenguaje juvenil español y chileno. En M. E. Placencia y C. García Fernández (eds.), Pragmática y comunicación intercultural en el mundo hispanohablante. London: Rodopi, 209-230.

Jørgensen, Annette y Stenstrøm, Anna-Brita. 2009. Dos marcadores pragmáticos contrastados en el lenguaje juvenil: El inglés like y el español como. Español Actual, 92, 103-121.

Kornfeld, Laura Malena. 2013. Atenuadores en la lengua coloquial argentina. Lingüística, 29, 17-49.

Labov, William. 1983. Modelos sociolingüísticos. Madrid: Cátedra.

Landone, Elena. 2009. Los marcadores del discurso y la cortesía verbal en español. Bern: Peter Lang.

Lázaro, Fernando. 1980. Estudios de lingüística. Barcelona: Crítica.

Lázaro, Fernando. 1997. El dardo en la palabra. Barcelona: Galaxia Gutenberg.

Montecino, Lésmer. 2004. Estrategias de intensificación y de atenuación en la conversación coloquial de jóvenes chilenos. Onomázein, 10, 9-32.

Montes, José Joaquín. 1980-1981. Sobre el como de atenuación. Boletín de Filologia, XXXI, 667-675.

Moreno, Antonio. 1992. Sintaxis y semántica de "como". Málaga: Librería Ágora. Moreno, Francisco. 2009. Principios de sociolingüistica y sociología del lenguaje. Barcelona: Ariel. 
Puga, Juana. 1997. La atenuación en el castellano de Chile: Un enfoque pragmalinguístico. Valencia: Estudios Iberoamericanos.

Puga, Juana. 2014. Cómo hablamos cuando hablamos. La atenuación en el castellano de Chile. Santiago: Ceibo ediciones.

Rabanales, Ambrosio. 1974. Queísmo y dequeísmo en el español de Chile. En Estudios filológicos y lingüísticos. Homenaje a Angel Rosenblat. Caracas: Instituto Pedagógico, 413-444.

San Martín, Abelardo. 2004-2005. Igual como marcador discursivo en el habla de Santiago de Chile: función pragmático-discursiva y estratificación social de su empleo. Boletín de Filología, XL, 201-232.

San Martín, Abelardo. 2011. Los marcadores interrogativos de control de contacto en el corpus PRESEEA de Santiago de Chile. Boletín de Filología, XLVI (2), 135-166.

San Martín, Abelardo. 2013. Los reformuladores de distanciamiento en el corpus PRESEEA de Santiago de Chile. Boletín de Filología, XLVIII(1), 171-199.

San Martín, Abelardo. 2015. Variantes y equivalentes funcionales de al final: los reformuladores de recapitulación en el habla santiaguina. RLA. Revista de Lingüistica Teórica y Aplicada, 53(2), 97-119.

San Martín, Abelardo. 2016a. Los marcadores de reformulación en el español oral de Santiago de Chile: análisis discursivo y sociolingüístico. Oralia, 19, 283-324.

San Martín, Abelardo. 2016b. Análisis sociolingüístico de los reformuladores de rectificación en el habla santiaguina. Literatura y Lingüistica, 33, 241-264.

San Martín, Abelardo y Guerrero, Silvana. 2013. Una aproximación sociolingüística al empleo del discurso referido en el corpus PRESEA de Santiago de Chile. Revista Signos. Estudios de lingüística, 46(82), 258-282.

San Martín, Abelardo y Guerrero, Silvana. 2015. Estudio sociolingüístico del español de Chile (ESECH): recogida y estratificación del corpus de Santiago. Boletín de Filología, L(1), 221-247.

Serrano, María José. 2011. Sociolingüística. Barcelona: Ediciones del Serbal.

Tagliamonte, Sali. 2009. Be like: The New Quotative in English. En N. Coupland y A. Jaworski (eds.), The New Sociolinguistics Reader. New York: Palgrave Macmillan, 75-91.

Tagliamonte, Sali. 2016. Teen Talk. The Language of Adolescents. Cambridge: Cambridge University Press.

Tannen, Deborah. 1991. Tú no me entiendes. Buenos Aires: Javier Vergara Editor. Vigara, Ana María. 1992. Morfosintaxis del español coloquial: esbozo estilístico. Madrid: Gredos. 